\title{
Antimicrobial resistance trends among important clinical pathogens reported from the ARINCQ surveillance of bacterial resistance, 2012-2018: multi-center retrospective surveillance study in Chongqing
}

\section{Shan Sun}

the first affiliated hospital of Chongqing Medical University

\section{Xiaolang Tian}

University of the Chinese Academy of Sciences

\section{Li Yan}

Chongqing Medical University First Affiliated Hospital

Jide Sun

Chongqing Medical University First Affiliated Hospital

\section{Chuanming Zhang}

Chongqing Medical University First Affiliated Hospital

Xiuyu Xu

Chongqing Medical University First Affiliated Hospital

\section{Chunmei Jing}

Chongqing Medical University Affiliated Children's Hospital

\section{Yunying Wang}

Chongqing Medical University Second Affiliated Hospital

\section{Wei Chen}

Three Gorges Hospital affiliated to Chongqing University

\section{Zhongzheng Xiong}

Dianjiang People's Hospital of Chongqing

\section{Xin Yao}

Banan people's hospital

\section{Minjun Zhang}

The people's Hospital of Jiulongpo District, Chognqing

Wen Qi

The people's Hospital of Kaizhou District, Chongqing

\section{Renyan Wang}

Chognqing Jiangbei district people's hospital

\section{Shulin You}

The people's hospital of Nanchuan district, Chongqing

\section{Yong Wu}

Fengdu People's hospital of Chongqing

\section{Mei Zhang}

The people's hospital of Qijiang District, Chongqing

\section{Xiaofeng Yu}

Traditional Chinese Medicine Hospital of Dianjiang, Chongqing

\section{Guangde Pei}

The people's hospital of Shapingba District, Chongqing

Juan Fang 
The people's hospital of Yubei district, Chongqing

\section{Zhongxiu Huang}

Qianjiang central hospital of Chongqing

\section{Ji Zhang}

The people's hospital of Changchou district, Chongqing

\section{Yuan Gao}

Jiangjin center hospital of Chongqing

\section{Gang Fu}

Chongqing University cancer hospital

\section{Niqi Xie}

The Dazu district people's hospital of Chongqing

\section{Zhen Ruan}

The ninth people's hospital of Chongqing

\section{Wenzhang Long}

Yongchuan Hospital of Chongqing Medical University

\section{Yuan Xiong}

Chongqing traditional Chinese Medicine Hospital

\section{Lijuan Li}

Chongqing University central hospital

\section{Lei Chen}

The people's hospital of Tongliang district, Chongqing

\section{Wenping Deng}

Fuling center hospital of Chongqing

\section{Xiaoli Ye}

The fifth people's hospital of Chongqing

\section{Chengfeng Shu}

Bishan hospital of Chongqing

\section{Yan Shen}

Chongqing Health Center for Women and Children

\section{Siqiang Niu}

Chongqing Medical University First Affiliated Hospital

Shifeng Huang ( $\square$ sfhuang@hospital.cqmu.edu.cn )

Chongqing Medical University First Affiliated Hospital

\section{Research}

Keywords: antimicrobial resistance, surveillance, multi-center, carbapenems, Southwestern China, carbapenem-resistant K. pneumonia

Posted Date: April 7th, 2020

DOl: https://doi.org/10.21203/rs.3.rs-20365/v1

License: (c) (i) This work is licensed under a Creative Commons Attribution 4.0 International License. Read Full License 


\section{Abstract}

Background: Data on the Antimicrobial resistance (AMR) trends of the important clinical isolates in southwestern China is lacking. This study was conducted to monitor the temporal AMR trends of the most common pathogens contributing to the majority of human infections.

Methods: A multi-center retrospective surveillance study was conducted from 2012 to 2018 . Antimicrobial susceptibility testing was carried out according to unified protocols using the Kirby-Bauer method or automated systems. Results were analyzed according to Clinical and Laboratory Standards Institute (CLSI) 2018 definitions. Data from the China Antimicrobial Resistance Surveillance System (CARSS) in Chongqing (ARINCQ) was analyzed by WHONET 5.6 software.

Results: The total number of bacterial isolates was between 49,636 and 128,460 annually. While the isolation rate of Acinetobacter baumannii (A. baumannii) presented decreasing trend, that of Staphylococcus aureus (S. aureus) showed increasing tendency. Escherichia coli (E. coli) showed decreasing susceptibility trends to most antibiotics except carbapenems. Resistance rates of Klebsiella pneumoniae (K. pneumonia) to ceftazidime (CAZ), ceftriaxone (CRO), and cefepime (FEP) are decreasing over time. Pseudomonas aeruginosa (P. aeruginosa) and $S$. aureus demonstrated obvious declining resistance trends to most of the antibiotics tested. Resistance of Enterococcus faecalis (E. faecalis) and Enterococcus faecium (E. faecium) to high-level gentamycin are decreasing continuously over time. The resistance rate of $A$. baumannii to meropenem increased from $48.3 \%$ to $57.7 \%$. The isolation rate of carbapenem-resistant K. pneumonia (CRKPN) increased annually from $2.6 \%$ in 2012 to $6.1 \%$ in 2018 . For CRKPN, consistent increasing trends in isolation rates were witnessed in both the children and the elderly groups, with its isolation rate in children being obviously higher than that in the elderly group from 2015 to 2018.

Conclusions: The prevalence of CRKPN has been significantly increased in both the children and the elderly groups ever since 2012, which calls for continuous resistance surveillance, colonization screening and clearance, environmental monitoring, and effective antimicrobial strategies. While carbapenems are still active against $K$. pneumonia and E. coli, vancomycin or linezolid is still effective against $S$. aureus and Enterococcus spp. Notably, this is the first report of an CRKPN isolate co-harboring triple carbapenemase genes including $b / a_{\mathrm{KPC}-2}, b / a_{\mathrm{NDM}-1}$ and $b / a_{\mathrm{IMP}-4}$ worldwide.

Keywords: antimicrobial resistance, surveillance, multi-center, carbapenems, Southwestern China, carbapenem-resistant $K$. pneumonia

\section{Background}

The China Antimicrobial Resistance Surveillance System (CARSS) (http://www.carss.cn/) covers all the provinces and autonomous regions in China. As Chongqing is the largest autonomous region in southwestern China and its demographic structure of urbanrural dualization is similar to that of the whole China, making the data of the antimicrobial resistance (AMR) in Chongqing representative in China. With the aim of gathering temporal trends on bacterial epidemiology and resistance from multiple laboratories in Chongqing, the Antimicrobial Resistance Surveillance Network in Chongqing (ARINCQ), a branch of CARSS, was organized in 2011, when there were only seven teaching hospitals. To prevent geographical bias, we successively enrolled hospitals in a geographical representative manner, and 59 hospitals have been enrolled in ARINCQ, almost covering all the regions and districts of Chongqing.

Worldwide AMR changing tendencies were reported one after another $[1,2,3,4]$, and the comprehensive and overall data of temporal AMR shifts of important clinical isolates in China was only reported by CHINET, which just included seventeen teaching hospitals $[1,5]$. We searched pubmed with the terms "antimicrobial resistance of nosocomial pathogenes", and the retrieval results consisted of larger national surveillance studies, reviews, or single case studies, with no results of the studies including comprehensive Chongqing coverage. In addition, the longitudinal province-level AMR surveillance in China was limited, and was mostly about the AMR changing trends of isolates from bloodstream and intra-abdominal infections $[6,7,8,9,10]$. The overall annual AMR changing trends over long time periods are still lacking in southwestern China. The present study was initiated to provide clear and comprehensive AMR changing trends for the important nosocomial pathogens from 2012 to 2018 in southwestern China, and meanwhile to deeply explore into the cause of the key problems discovered. 


\section{Methods}

\section{Data enrollment criteria}

All bacterial data including patients' information, bacterial identification and antimicrobial susceptibility from 59 microbiological laboratories in Chongqing, covering secondary and tertiary hospitals with a ratio of 1.5:1, were acquired from CARSS database in Chongqing (ARINCQ) between 2012 and 2018. Children were defined as patients no older than 14-year-old, adults were defined as patients between 15 and 65 years old, while the elderly were defined as patients older than 65 years old.

Only the first isolate from each individual patient was included in this study.

\section{Bacterial identification and antimicrobial susceptibility test}

All the isolates were identified at the species level by semi- or automated systems, such as the VITEK2 compact (bioMérieux, Inc., Durham, NC) system, or the VITEK MS (bioMerieux, Hazelwood, MO, United States) automated system, and so on. Routine antimicrobial susceptibility testings were performed by using either the API system, the BD Phoenix System (Becton Dickinson, America), or the VITEK2 compact (bioMérieux, Inc., Durham, NC) system. According to the breakpoint recommendations by the Clinical and Laboratory Standards Institute, 2018 (CLSI-2018), K. pneumoniae or E. coli isolates which were resistant to at least one of the carbapenems, with the criteria of minimum inhibitory concentration (MIC) of $\geq 2 \mu \mathrm{g} / \mathrm{mL}$ for ertapenem, $\geq 4 \mu \mathrm{g} / \mathrm{mL}$ for imipenem, or $\geq 4 \mu \mathrm{g} / \mathrm{mL}$ for meropenem, were defined as carbapenem-resistant Enterobacteriaceae (CRE); while carbapenemresistant $A$. baumannii (CRABA) or $P$. aeruginosa (CRPAE) strains were defined as $\mathrm{MIC} \geq 8 \mu \mathrm{g} / \mathrm{mL}$ for imipenem or meropenem.

\section{Detection of Carbapenemase genes}

Polymerase chain reaction was used to detect the potential presence of carbapenemase genes including $b / a_{\mathrm{KPC}}, b / a_{\mathrm{NDM}}, b / a_{\mathrm{VIM}}$, $b / a_{\mathrm{IMP}}, b / a_{\mathrm{GES}}, b / a_{\mathrm{OXA}-48-\mathrm{like}}$ and $b / a_{\mathrm{OXA}-181-\text { like, }}$ and all the variants of these carbapenemase genes were confirmed by sequencing. Moreover, the Carba NP test and SCIM were performed on all isolates to determine whether any bacteria produced carbapenemases by phenotypic methods but were negative by genotypic methods, or vice versa [11].

\section{Statistical analyses}

Raw data was firstly processed by WHONET 5.6 software (version 5.6, http://www.whonet.org/ software.html) and then analyzed on SPSS v.23.0 (SPSS, Chicago, IL, USA) software. The changing trends in AMR of each species and in the isolation rates of the main resistance phenotypes over the study period were determined using the linear trend analysis method. A statistically significant trend was established if the $P$ value was $<0.05$. The $P$ value was provided whenever there was a significant trend in the resistance. Moreover, the regression coefficient was calculated and indicated the change in the percentage of resistant isolates (on the $y$-axis) over time (on the x-axis) $[12,13]$. A negative slope (-) indicated a decrease in resistance over time, whereas a positive slope (+) presented an increase in resistance over time. The difference in AMR between children and non-children was further assessed by Pearson Chi-square test. Statistical significance was confirmed if a two-tailed P value was no more than 0.05.

\section{Results}

\section{Changing trends of the isolation percentages of the main clinical pathogen species}

Between 2012 and 2018, the number of non-repetitive bacterial isolates ranged between 49,636 and 128,460 annually. The ratio of specimen types during the study period did not change. The percentages of the seven investigated species (E. coli, K. pneumoniae, $P$. aeruginosa, $A$. baumannii, $S$. aureus, E. faecium, and E. faecalis) among total isolates were shown in Table 1 . While the annual total percentage of the seven pathogen species among the total isolates didn't change much, accounting from 60.2 to $63.4 \%$, the percentage of $A$. baumannii showed a slowly decreasing trend, whereas the ratio of $S$. aureus presented a slowly increasing tendency (Table 1).

Table 1 Percentages of the main clinical pathogens among total clinical isolates 


\begin{tabular}{|c|c|c|c|c|c|c|c|c|c|c|c|c|c|c|}
\hline \multicolumn{2}{|c|}{ E.coli } & \multicolumn{2}{|c|}{ K.pneumoniae } & \multicolumn{2}{|c|}{ P.aeruginosa } & \multicolumn{2}{|c|}{ A.baumannii } & \multicolumn{2}{|c|}{ S.aureus } & \multicolumn{2}{|c|}{ E.faecalis } & \multicolumn{2}{|c|}{ E.faecium } & \multirow{2}{*}{$\begin{array}{l}\text { Total percentage } \\
\text { among all }\end{array}$} \\
\hline $\mathrm{n}$ & $\%$ & $\mathrm{n}$ & $\%$ & $\mathrm{n}$ & $\%$ & $\mathrm{n}$ & $\%$ & $\mathrm{n}$ & $\%$ & $\mathrm{n}$ & $\%$ & $\mathrm{n}$ & $\%$ & \\
\hline 9806 & 19.8 & 6520 & 13.1 & 4326 & 8.7 & 3650 & 7.4 & 4039 & 8.1 & 1054 & 2.1 & 886 & 1.8 & 61.0 \\
\hline 15931 & 19.2 & 12513 & 15.1 & 7264 & 8.7 & 6348 & 7.6 & 6727 & 8.1 & 1539 & 1.9 & 1451 & 1.7 & 62.3 \\
\hline 18784 & 19.7 & 13566 & 14.2 & 7374 & 7.7 & 6122 & 6.4 & 8608 & 9 & 1546 & 1.6 & 1546 & 1.6 & 60.2 \\
\hline 19714 & 20.9 & 13753 & 14.6 & 7650 & 8.1 & 5523 & 5.8 & 8815 & 9.3 & 1811 & 1.9 & 1601 & 1.7 & 62.3 \\
\hline 20408 & 21.7 & 13107 & 13.9 & 7752 & 8.2 & 5145 & 5.5 & 9456 & 10.1 & 1866 & 2.0 & 1846 & 2.0 & 63.4 \\
\hline 21996 & 21.0 & 14464 & 13.8 & 8549 & 8.2 & 5665 & 5.4 & 10862 & 10.4 & 2176 & 2.1 & 2133 & 2.0 & 62.9 \\
\hline
\end{tabular}

\section{BACTERIAL RESISTANCE AND TRENDS IN GRAM-NEGATIVE BACILLI}

\section{E. coli and K. pneumoniae}

Statistically significant decreases in AMRs of E.colito nine (75\%) out of the 12 tested antibiotics were observed between 2012 and 2018 , which included SAM (from $51.2 \%$ to $40.8 \%, P<0.001$ ), SCF (13.0\% to $4.8 \%, P<0.001$ ), TZP ( $4.8 \%$ to $2.9 \%, P<0.001)$, CAZ (29.7\% to $19.6 \%, P<0.001)$, CRO (65.3\% to $49.1 \%, P<0.001)$, FEP (33.4\% to $22.6 \%, P<0.001)$, AMK ( $3.4 \%$ to $1.6 \%, P<0.001), \operatorname{LVX}(47.1 \%$ to $40.3 \%, P<0.001)$, and SXT $(62.8 \%$ to $52.7 \%, P<0.001)$. Its resistance rates to IPM and MEM increased from $0.7 \%$ to $1.9 \%$ and $0.6 \%$ to $1.4 \%$ from 2012 to 2016 , but then decreased to $1.0 \%$ and $0.9 \%$ in 2018 , respectively. However, the overall resistance rates to IPM and MEM showed significant statistical increases over the study period $(P<0.001$ and $P<0.001)$. The FOX resistance rates fluctuated around $12.0 \%(P=0.164)$ (Table 2$)$.

Table 2. Resistance rates (\%) of E.coli to commonly used antibiotics

\begin{tabular}{|c|c|c|c|c|c|c|c|c|c|}
\hline Antibiotics & $\begin{array}{c}2012 \\
(n=9806)\end{array}$ & $\begin{array}{c}2013 \\
(n=15931) \\
\end{array}$ & $\begin{array}{c}2014 \\
(n=18784) \\
\end{array}$ & $\begin{array}{c}2015 \\
(n=19714)\end{array}$ & $\begin{array}{c}2016 \\
(n=20408)\end{array}$ & $\begin{array}{c}2017 \\
(n=21996)\end{array}$ & $\begin{array}{c}2018 \\
(n=26436)\end{array}$ & $P$ value & $\mathrm{RC}$ \\
\hline Ampicillin/Sulbactam & 51.2 & 46 & 44.5 & 44.4 & 45.1 & 43 & 40.8 & $<0.001$ & -0.881 \\
\hline Piperacillin/tazobactam & 4.8 & 3 & 3.3 & 3.1 & 3.6 & 2.9 & 2.9 & $<0.001$ & -0.638 \\
\hline Cefoperazone/sulbactam & 13.0 & 6.2 & 5.3 & 4.5 & 5.8 & 5.5 & 4.8 & $<0.001$ & -0.667 \\
\hline Ceftazidime & 29.7 & 26.6 & 23 & 22.7 & 22.0 & 19.6 & 19.6 & $<0.001$ & -0.949 \\
\hline Ceftriaxone & 65.3 & 62 & 55 & 54.1 & 52.2 & 51 & 49.1 & $<0.001$ & -0.951 \\
\hline Cefepime & 33.4 & 28.5 & 29.1 & 27.8 & 21 & 18.2 & 22.6 & $<0.001$ & -0.883 \\
\hline Cefoxitin & 13.3 & 9.2 & 9 & 12.5 & 11.9 & 11.2 & 10.7 & 0.164 & -0.043 \\
\hline Imipenem & 0.7 & 0.7 & 0.7 & 1.2 & 1.9 & 1.1 & 1.0 & $<0.001$ & +0.519 \\
\hline Meropenem & 0.6 & 0.6 & 0.6 & 1.4 & 1.4 & 0.7 & 0.9 & $<0.001$ & +0.399 \\
\hline Amikacin & 3.4 & 2.6 & 3.2 & 2.1 & 1.8 & 1.6 & 1.6 & $<0.001$ & -0.905 \\
\hline Levofloxacin & 47.1 & 44.6 & 40.1 & 38.3 & 38.0 & 39.9 & 40.3 & $<0.001$ & -0.727 \\
\hline Sulfamethoxazole/trimethoprim & 62.8 & 61.5 & 57.4 & 56.5 & 56.0 & 53.8 & 52.7 & $<0.001$ & -0.973 \\
\hline
\end{tabular}

The values in parentheses indicate the total isolated numbers of E.coli in each year; RC, regression coefficient. 
The overall resistance rates to all the antibiotics showed statistically significant changing trends over the study period. These antibiotics with increasing AMRs included IMP (from 1.8\% to 5.5\%, $P<0.001$ ), MEM (2.3\% to $5.0 \%, P<0.001$ ), SCF (from $6.7 \%$ to $11.8 \%, P<0.001$ ), FOX (from $14.1 \%$ to $18.2 \%, P<0.001$ ), AMK (from $2.9 \%$ to $5.2 \%, P<0.001$ ) and LVX (from $7.9 \%$ to $10.9 \%, P<0.001$ ). While the antibiotics with decreasing AMRs contained SAM (from $37.3 \%$ to $27.9 \%, P<0.001$ ), TZP (from $12.3 \%$ to $8.8 \%, P=0.003$ ), CAZ (from $24.2 \%$ to $15.7 \%, P<0.001$ ), CRO (33.7\% to $26.4 \%, P<0.001$ ), FEP (from $19.6 \%$ to $15 \%, P<0.001$ ) and SXT (from $31.9 \%$ to $22.1 \%, P<0.001)$ (Table 3).

Table 3. Resistance rates (\%) of $K$. pneumoniae to commonly used antibiotics

\begin{tabular}{|c|c|c|c|c|c|c|c|c|c|}
\hline Antibiotics & $\begin{array}{c}2012 \\
(n=6520)\end{array}$ & $\begin{array}{c}2013 \\
(n=12513) \\
\end{array}$ & $\begin{array}{c}2014 \\
(n=13566) \\
\end{array}$ & $\begin{array}{c}2015 \\
(n=13753) \\
\end{array}$ & $\begin{array}{r}2016 \\
(n=13107) \\
\end{array}$ & $\begin{array}{c}2017 \\
(n=14464) \\
\end{array}$ & $\begin{array}{c}2018 \\
(n=16882) \\
\end{array}$ & $P$ value & $\mathrm{RC}$ \\
\hline Ampicillin/Sulbactam & 37.3 & 29.7 & 27.9 & 26 & 29.3 & 29.4 & 27.9 & $<0.001$ & -0.586 \\
\hline Piperacillin/tazobactam & 12.3 & 6 & 5.9 & 5.8 & 6.9 & 8.1 & 8.8 & 0.003 & -0.175 \\
\hline Cefoperazone/sulbactam & 6.7 & 3.1 & 4.4 & 5.2 & 8.2 & 12.8 & 11.8 & $<0.001$ & +0.804 \\
\hline Ceftazidime & 24.2 & 18.5 & 15.2 & 15.2 & 15.9 & 15.5 & 15.7 & $<0.001$ & -0.719 \\
\hline Ceftriaxone & 33.7 & 33 & 24.2 & 23.8 & 26 & 27.7 & 26.4 & $<0.001$ & -0.592 \\
\hline Cefepime & 19.6 & 14.6 & 14.6 & 13.9 & 12.7 & 12.4 & 15 & $<0.001$ & -0.651 \\
\hline Cefoxitin & 14.1 & 8.8 & 10.2 & 10.9 & 12.6 & 16.3 & 18.2 & $<0.001$ & +0.675 \\
\hline Imipenem & 1.8 & 1.5 & 1.6 & 2.3 & 4.6 & 5.5 & 5.5 & $<0.001$ & +0.917 \\
\hline Meropenem & 2.3 & 1.5 & 1.4 & 2.8 & 3.9 & 4.5 & 5.0 & $<0.001$ & +0.892 \\
\hline Amikacin & 2.9 & 2.4 & 3.4 & 1.6 & 3.3 & 4.7 & 5.2 & $<0.001$ & +0.702 \\
\hline Levofloxacin & 7.9 & 7.2 & 6.9 & 6.9 & 8.4 & 9.6 & 10.9 & $<0.001$ & +0.78 \\
\hline Sulfamethoxazole/trimethoprim & 31.9 & 26.3 & 23.9 & 22.9 & 24.1 & 22.8 & 22.1 & $<0.001$ & -0.825 \\
\hline
\end{tabular}

The values in parentheses indicate the total isolated numbers of $K$. pneumoniae in each year; RC, regression coefficient.

\section{P. aeruginosa and baumannii complex}

The resistance rates of $P$. aeruginosa to all the eleven investigated antibiotics were declining with statistical significance. The AMR rates of $P$. aeruginosa to all the antibiotics tested were less than $26 \%$ during the study period and all were below $19 \%$ in 2018 (Table 4). 
Table 4. Resistance rates (\%) of $P$. aeruginosa to commonly used antibiotics

\begin{tabular}{|c|c|c|c|c|c|c|c|c|c|}
\hline Antibiotics & $\begin{array}{c}2012 \\
(n=4326)\end{array}$ & $\begin{array}{c}2013 \\
(n=7264)\end{array}$ & $\begin{array}{c}2014 \\
(n=7374)\end{array}$ & $\begin{array}{c}2015 \\
(n=7650)\end{array}$ & $\begin{array}{c}2016 \\
(n=7752)\end{array}$ & $\begin{array}{c}2017 \\
(n=8549 \square\end{array}$ & $\begin{array}{c}2018 \\
\square n=10381 \square\end{array}$ & $P$ value & $\mathrm{RC}$ \\
\hline Piperacillin & 24.4 & 22.7 & 18.2 & 15.7 & 15.8 & 13.4 & 13.4 & $<0.001$ & -0.953 \\
\hline Ticarcillin/Clavulanic acid & 24.9 & 25.3 & 22.4 & 19.8 & 21.2 & 21.5 & 16.4 & $<0.001$ & -0.869 \\
\hline Piperacillin/tazobactam & 13.3 & 14.6 & 10.9 & 9.5 & 10.6 & 9.3 & 9.9 & $<0.001$ & -0.804 \\
\hline Ceftazidime & 15.4 & 17.8 & 15.7 & 13.2 & 13.5 & 12.2 & 12.7 & $<0.001$ & -0.826 \\
\hline Cefepime & 12.5 & 14.3 & 12.7 & 11 & 10.2 & 9.5 & 11 & $<0.001$ & -0.775 \\
\hline Aztreonam & 19.1 & 18.7 & 16.8 & 15.8 & 16 & 15.7 & 18.4 & 0.020 & -0.465 \\
\hline Imipenem & 20.2 & 15.7 & 15.1 & 13.7 & 13.9 & 12.5 & 12.3 & $<0.001$ & -0.896 \\
\hline Meropenem & 18.2 & 14.4 & 12.7 & 11.7 & 11.4 & 9.7 & 10 & $<0.001$ & -0.925 \\
\hline Ciprofloxacin & 18.4 & 14.7 & 13.1 & 11.2 & 10.1 & 9.1 & 10.5 & $<0.001$ & -0.902 \\
\hline Levofloxacin & 16.4 & 13.3 & 11.5 & 10.2 & 9.3 & 9 & 10.6 & $<0.001$ & -0.834 \\
\hline Amikacin & 10.5 & 9.5 & 7.8 & 4.9 & 4.5 & 3.1 & 3.3 & $<0.001$ & -0.965 \\
\hline
\end{tabular}

The values in parentheses indicate the total isolated numbers of P.aeruginosa in each year; RC, regression coefficient.

For $A$. baumannii complex, while its resistance rates to MEM (from $48.3 \%$ to $57.7 \%, P<0.001$ ), IMP (from $52.1 \%$ to $55.4 \%, P<0.001$ ), TZP (from $48.0 \%$ to $56.5 \%, P<0.001$ ) and LVX (from $36.9 \%$ to $47.2 \%, P<0.001$ ) annually increased from 2012 to 2018 , its resistance rates to CAZ, AMK and MNO decreased from $64.1 \%$ to $57 \%(P<0.001), 55.7 \%$ to $46.5 \%(P<0.001)$ and $19.5 \%$ to $14.1 \%(P<0.001)$, respectively. There were no continuous changing trends for FEP and PIP, with their respective resistance rates fluctuating between $55.5 \%$ and $59.2 \%(P=0.490)$, and $52.5 \%$ and $70.6 \%(P=0.952)$ (Table 5$)$. 
Table 5. Resistance rates (\%) of $A$. baumannii to commonly used antibiotics

\begin{tabular}{cccccccccccccccc}
\hline & 2012 & 2013 & 2014 & 2015 & 2016 & 2017 & 2018 & \\
Antibiotics & $(\mathrm{n}=3650)$ & $(\mathrm{n}=6348)$ & $(\mathrm{n}=6122)$ & $(\mathrm{n}=5523)$ & $(\mathrm{n}=5145)$ & $(\mathrm{n}=5665)$ & $(\mathrm{n}=6703)$ & $P$ value & $\mathrm{RC}$ \\
\hline Piperacillin & 52.5 & 64.9 & 61.9 & 64.8 & 70.6 & 58 & 58.6 & 0.952 & +0.173 \\
\hline Piperacillin/tazobactam & 48 & 54.1 & 49.2 & 53.5 & 58.6 & 54.9 & 56.5 & $<0.001 \quad+0.744$ \\
\hline Ceftazidime & 64.1 & 61.6 & 56.9 & 59.9 & 60.3 & 56.5 & 57 & $<0.001 \quad-0.764$ \\
\hline Cefepime & 56.8 & 58 & 55.5 & 57.9 & 59.2 & 55.7 & 56.6 & 0.490 & -0.087 \\
\hline Imipenem & 52.1 & 52.7 & 48.2 & 53.2 & 51.7 & 51.3 & 55.4 & $<0.001 \quad+0.375$ \\
\hline Meropenem & 48.3 & 53.3 & 51.5 & 57.4 & 62.4 & 55.2 & 57.7 & $<0.001 \quad+0.717$ \\
\hline Levofloxacin & 36.9 & 34.6 & 33.6 & 44.6 & 44.6 & 43.1 & 47.2 & $<0.001 \quad+0.829$ \\
\hline Amikacin & 55.7 & 48.2 & 44.2 & 44.7 & 50.9 & 46.2 & 46.5 & $<0.001 \quad-0.474$ \\
\hline Minocycline & 19.5 & 25.2 & 12.3 & 29.2 & 22.4 & 12.5 & 14.1 & $<0.001$ & -0.366 \\
\hline
\end{tabular}

The values in parentheses indicate the total isolated numbers of $A$. baumannii in each year; RC, regression coefficient.

\section{BACTERIAL RESISTANCE AND TRENDS IN GRAM-POSITIVE COCCI}

\section{S. aureus}

Evident annual decreasing AMR trends were observed for $S$. aureus to 10 of $13(76.9 \%)$ investigated antibiotics comprising GEN (from $28.9 \%$ to $11.2 \%$ ), RIF (from $10.3 \%$ to $2 \%$ ), LVX (from $28.6 \%$ to $9.5 \%$ ), SXT (from $29.5 \%$ to $13.9 \%$ ), ERY (from $64.1 \%$ to $57.6 \%$ ), CLI (from $43 \%$ to $37.4 \%$ ) , TET (from $33.3 \%$ to $22 \%$ ) , PEN(from $95.9 \%$ to $93.7 \%$ ) and OXA (from $26.9 \%$ to $24.6 \%$ ). No LNZ-, VAN- or TEC-resistant $S$. aureus isolates were observed during the study period (Table 6). 
Table 6. Resistance rates (\%) of $S$. aureus to commonly used antibiotics

\begin{tabular}{|c|c|c|c|c|c|c|c|c|c|}
\hline Antibiotics & $\begin{array}{c}2012 \\
(n=4039)\end{array}$ & $\begin{array}{c}2013 \\
(n=6727) \\
\end{array}$ & $\begin{array}{c}2014 \\
(n=8608)\end{array}$ & $\begin{array}{c}2015 \\
(\mathrm{n}=8815) \\
\end{array}$ & $\begin{array}{c}2016 \\
(n=9456) \\
\end{array}$ & $\begin{array}{c}2017 \\
(n=10862) \\
\end{array}$ & $\begin{array}{c}2018 \\
(n=12977) \\
\end{array}$ & $P$ value & $\mathrm{RC}$ \\
\hline Penicillin G & 95.9 & 95.6 & 96.3 & 95.9 & 94.8 & 93.6 & 93.7 & $<0.001$ & -0.848 \\
\hline Oxacilli & 26.9 & 27.2 & 29.4 & 28.2 & 26.2 & 25.9 & 24.6 & $<0.001$ & -0.624 \\
\hline Gentamicin & 28.9 & 24.5 & 22.8 & 19.8 & 13.2 & 12.1 & 11.2 & $<0.001$ & -0.979 \\
\hline Rifampin & 10.3 & 8.3 & 6.7 & 5 & 2.4 & 2.2 & 2 & $<0.001$ & -0.971 \\
\hline Levofloxacin & 28.6 & 23.6 & 19 & 15.5 & 11.6 & 10.3 & 9.5 & $<0.001$ & -0.973 \\
\hline Sulfamethoxazole/trimethoprim & 29.5 & 26.8 & 23.5 & 22 & 19.8 & 15.4 & 13.9 & $<0.001$ & -0.994 \\
\hline Azithromycin & 65.8 & 65.4 & 66.7 & 64.6 & 62.1 & 65.5 & 63.3 & $<0.001$ & -0.580 \\
\hline Clindamycin & 43 & 43.9 & 43.1 & 44.8 & 41.3 & 36.7 & 37.4 & $<0.001$ & -0.796 \\
\hline Erythromycin & 64.1 & 63.2 & 63.2 & 61.2 & 59.6 & 58.9 & 57.6 & $<0.001$ & -0.983 \\
\hline Tetracycline & 33.3 & 29.2 & 27.4 & 25.2 & 24.9 & 22.3 & 22 & $<0.001$ & -0.967 \\
\hline Linezolid & 0 & 0 & 0 & 0 & 0 & 0 & 0 & - & - \\
\hline Vancomycin & 0 & 0 & 0 & 0 & 0 & 0 & 0 & - & - \\
\hline Teicoplanin & 0 & 0 & 0 & 0 & 0 & 0 & 0 & - & - \\
\hline
\end{tabular}

The values in parentheses indicate the total isolated numbers of $S$. aureus in each year; The horizontal line indicates no statistical analysis; $\mathrm{RC}$, regression coefficient.

\section{E. faecalis and E. faecium}

The resistance rates of $E$. faecalis to VAN fluctuated around $0.3 \%(P=0.163)$. While the resistance rates to LNZ showed decreasing trend over time $(P=0.009)$. Quinolone resistance rates only showed a slight change with no statistical significance. Its resistance rates to GEH and ERY respectively declined from $49.8 \%$ to $38.6 \%(P<0.001)$ and from $74.4 \%$ to $66.9 \%(P<0.001)$. Its resistance rate to AMP maintained below $7.4 \%$ during the surveillance periods (Table 7). On the contrary, the resistance rates of E. faecium to AMP remained higher above $82 \%$ during the study period with no obvious change $(P=0.871)$. Obvious continuous decrease of resistance rate to GEH and ERY was observed from $69.8 \%$ to $50.7 \%(P<0.001)$ and $89.1 \%$ to $85.6 \%(P=0.001)$, respectively. E. faecium was highly resistant to quinolones with resistance rate of higher than $79 \%$ during the study period. While its resistance rates to both LNZ and VAN showed declining trend from $0.8 \%$ to $0.4 \%(P=0.019)$ and $1.2 \%$ to $0.8 \%(P<0.001)$ between 2012 and 2018 , respectively (Table 8). 
Table 7. Resistance rates (\%) of $E$. faecalis to commonly used antibiotics

\begin{tabular}{cccccccccc} 
& 2012 & 2013 & 2014 & 2015 & 2016 & 2017 & 2018 \\
Antibiotics & $(\mathrm{n}=1054)$ & $(\mathrm{n}=1539)$ & $(\mathrm{n}=1546)$ & $(\mathrm{n}=1811)$ & $(\mathrm{n}=1866)$ & $(\mathrm{n}=2176)$ & $(\mathrm{n}=2569$ & $P$ value & $\mathrm{RC}$ \\
\hline Ampicillin & 5.2 & 6.6 & 7.4 & 6.4 & 4 & 5.2 & 4.6 & 0.001 & -0.511 \\
\hline Gentamicin-High & 49.8 & 42.2 & 45.3 & 43 & 43.4 & 40.8 & 38.6 & $<0.001$ & -0.831 \\
\hline Levofloxacin & 24.7 & 23.5 & 23.5 & 23.3 & 20.7 & 22.1 & 23.6 & 0.238 & -0.534 \\
\hline Ciprofloxacin & 25.4 & 27.3 & 27.9 & 26.6 & 23.3 & 26.7 & 27.5 & 0.869 & +0.024 \\
\hline Moxifloxacin & 21 & 22.3 & 21.4 & 20.7 & 17.9 & 17.4 & 22.9 & 0.362 & -0.279 \\
\hline Erythromycin & 74.4 & 73.4 & 72.2 & 69.6 & 68.7 & 69.5 & 66.9 & $<0.001$ & -0.960 \\
\hline Tetracycline & 82.5 & 77.2 & 77.5 & 78.8 & 79.6 & 80.7 & 78.9 & 0.730 & -0.071 \\
\hline Linezolid & 1.7 & 3.4 & 3.5 & 2.5 & 2.1 & 1.4 & 2.2 & 0.009 & -0.376 \\
\hline Vancomycin & 0.3 & 0.3 & 0.3 & 0.5 & 0.3 & 0.1 & 0.2 & 0.163 & -0.445 \\
\hline
\end{tabular}

The values in parentheses indicate the total isolated numbers of E. faecalis in each year; RC, regression coefficient.

Table 8. Resistance rates (\%) of $E$. faecium to commonly used antibiotics

\begin{tabular}{cccccccccc} 
& 2012 & 2013 & 2014 & 2015 & 2016 & 2017 & 2018 & \\
Antibiotics & $(\mathrm{n}=886)$ & $(\mathrm{n}=1451)$ & $(\mathrm{n}=1546)$ & $(\mathrm{n}=1601)$ & $(\mathrm{n}=1846)$ & $(\mathrm{n}=2133)$ & $(\mathrm{n}=2614)$ & $P$ value & $\mathrm{RC}$ \\
\hline Ampicillin & 88.1 & 82.8 & 82.3 & 83.3 & 84.2 & 84.1 & 84.3 & 0.871 & -0.281 \\
\hline Gentamicin-High & 69.8 & 64.2 & 60.2 & 58.2 & 57.1 & 52.6 & 50.7 & $<0.001$ & -0.981 \\
\hline Levofloxacin & 82.3 & 79.1 & 81.1 & 83 & 84.2 & 81.8 & 80.9 & 0.518 & +0.203 \\
\hline Ciprofloxacin & 84.7 & 85.8 & 86.6 & 86.8 & 86.5 & 85.1 & 83.4 & 0.032 & -0.337 \\
\hline Moxifloxacin & 90.2 & 88.8 & 87.2 & 87.1 & 90.5 & 88.7 & 87.2 & 0.243 & -0.317 \\
\hline Erythromycin & 89.1 & 87.4 & 88 & 86 & 86.9 & 85 & 85.6 & 0.001 & -0.880 \\
\hline Tetracycline & 59.4 & 56.2 & 56.7 & 58.5 & 64.1 & 60 & 60.4 & 0.003 & +0.526 \\
\hline Linezolid & 0.8 & 0.5 & 1 & 0.5 & 0.4 & 0.3 & 0.4 & 0.019 & -0.677 \\
\hline Vancomycin & 1.2 & 2.4 & 2.9 & 1.8 & 1.4 & 0.7 & 0.8 & $<0.001$ & -0.575 \\
\hline
\end{tabular}

The values in parentheses indicate the total isolated numbers of E. faecium in each year; RC, regression coefficient.

\section{Changing trends of the main resistance phenotypes for the key pathogen species}

\section{MRSA and VRE}


The isolation rates of MRSA and VREFM were under $30 \%$ and $3 \%$, respectively, with a respective increase of $2.5 \%$ and $1.7 \%$ from 2012 to 2014 firstly, and a decrease of $4.8 \%$ and $2.1 \%$ respectively from 2014 to 2018 . And both the isolation rates of MRSA and VREFM showed declining trends over time $(P<0.001)$. VREFA isolation rates fluctuated around $0.3 \%(P=0.163)$ (Figure 1$)$.

\section{CRE, CRABA and CRPAE}

CRKPN isolation rates increased annually from $2.6 \%$ in 2012 to $6.1 \%$ in $2018(P<0.001)$. Although both of the CRECO and CRABA isolation rates presented increasing trends from 2012 to 2018 ( $P=0.016$ and $P<0.001$, respectively), the isolation rates of CRABA maintained stable from 2013 to 2018 ( $P=0.673$ ). The isolation rate of CRECO also declined continuously for the recent two years, remaining at a low level of $1.3 \%$. CRPAE isolation rates decreased annually from $23.8 \%$ in 2012 to $15.2 \%$ in $2018(P<0.001)$ (Figure2).

\section{Isolation rates of CRKPN and CRECO according to different age groups}

As for CRKPN isolation rates, all the three groups witnessed statistically annual increasing trends over time $(P<0.001)$ : from $2.8 \%$ (35/1252) in 2012 to $10.9 \%$ (236/2169) in 2018 in the children group $(P<0.001)$; from 2.8\% (88/3135) in 2012 to 4.4\% (303/6896) in 2018 in the adults group $(P<0.001)$; from 1.5\% (33/2133) in 2012 to 6.3\% (489/7817) in 2018 in the adults group $(P<0.001)$. The isolation rates of CRKPN in the children group presented a sharp increase and have been obviously higher than those in the nonchildren groups ever since 2015 (2015, 5.8\% vs. $2.6 \%, P<0.001 ; 2016,8.0 \%$ vs. $5.0 \%, P<0.001 ; 2017,9.5 \%$ vs. $5.5 \%, P<0.001 ; 2018$, $10.9 \%$ vs. $5.4 \%, P<0.001$ ) (Figure 3 ).

As for CRECO isolation rates, while there was no consistent changing tendency in the elderly group $(P=0.202)$, there was a significant declining trend in the adults group $(P<0.001)$ during the study period. For the children group, however, there was a consistent statistically increasing trend from $0.6 \%$ in 2012 to $6.4 \%$ in $2016(P<0.001)$, and then it dropped surprisingly from $6.4 \%$ in 2016 to $4.2 \%$ in 2018. Furthermore, the CRECO isolation rates in children have been remarkably higher than those in the nonchildren groups ever since 2015 (2015, 2.7\% vs.1.7\%, $P<0.001 ; 2016,6.4 \%$ vs. $1.4 \%, P<0.001 ; 2017,4.4 \%$ vs. $0.8 \%, P<0.001 ; 2018$, $4.2 \%$ vs. $1.0 \%, P<0.001$ ) (Figure 4 ).

\section{Distribution of carbapenemase genes from the CRKPN isolates}

Carbapenemase-producing Enterobacteriaceae (CPE) is the most pervasive antibiotic resistance threat to health services worldwide. Few alternative antibiotics (eg, coloistin, fosfomycin, and tigecycline) remain, and what's more, resistance can extend even to agents still in development or recently approved. Thus, public health efforts are beginning to emphasize containment of CPE in populations and health-care networks. This requires an understanding of the geographical distribution of CPE infections, their population reservoirs, and the risk factors for acquisition. However, there is little internationally comparable data.

In the present study, a total of 79 CRKPN isolates from 17 hospitals covering all the five geographical areas in Chongqing were collected during 2015-2016. 84.8\% of the CRKPN isolates harbored carbapenemase genes, with $b / a_{\mathrm{KPC}}, b / a_{\mathrm{NDM}}$ and $b / a_{\mathrm{IMP}}$ accounting for $51.9 \%(41 / 79), 39.2 \%$ (31/79) and $5 \%$ (4/79), respectively. bla $a_{\mathrm{VIM}}, b / a_{\mathrm{GES}}$, and bla $a_{\mathrm{OXA}}$ were not detected in this study. $11.4 \%(9 / 79)$ of the strains harbored two or three types of carbapenemase genes $\left(b / a_{\mathrm{KPC}-2}\right.$ and $b / a_{\mathrm{NDM}-1}, \mathrm{n}=7 ; b / a_{\mathrm{KPC}-2}$ and $b / a_{\mathrm{IMP}-4}$, $\mathrm{n}=1 ; b / a_{\mathrm{KPC}-2}, b / a_{\mathrm{NDM}-1}$ and $\left.b / a_{\mathrm{IMP}-4}, \mathrm{n}=1\right)$. The distribution map of carbapenemase genes in Chongqing was shown in Figure 5 .

\section{Discussion}

While a nationwide multi-center study in Korea showed increased AMRs to 3rd CEPs both for ECO and KPN from 2004 to 2012 [14], our study demonstrated gradual decreasing resistance tendencies for most antibiotics including third and fourth-generation cephalosporins ( $3^{\text {rd }}$ and $4^{\text {th }}$ CEPs), which was congruent with a previous report from CHINET in China which declared decreasing resistance trends to $3^{\text {rd }}$ and $4^{\text {th }}$ CEPs for ECO and KPN during 2012-2017 [1].

The emergence of CRE raises a global health-care threat. CRE infections are related to high mortality because therapeutic options are very limited $[15,16,17,18]$. As to the isolation rates of carbapenem-resistant Enterobateriaceae, that of CRKPN was much higher than that of CRECO according to Figure 2. Although the isolation rate of CRKPN in western China was lower compared with 
that from CHINET [1], the increasing CRKPN isolation rates are still noteworthy, especially more attention should be paid to the CRE isolated from children and the elders.

To further clarify the epidemiology of CRKPN in different patient age cohorts, the annual changing trends of isolation rates of CRKPN with a comparison with those of CRECO stratified by age were shown in Figure 3 and 4 . It could be concluded that the isolation rates of CRKPN in both the children and the elders increased consistently over time, which contributed significantly to continuous increasing annual CRKPN isolation rates. Notably, more attention should be paid to CRKPN in children its isolation rates in children were approximately twice higher than those in adults from 2015 to 2018.

Carbapenemase-producing Enterobacteriaceae (CPE) is the most pervasive antibiotic resistance threat to health services worldwide. The most globally widespread carbapenemase genes are typically carried on mobile genetic elements which can be freely exchanged between bacterial strains and species via horizontal gene transfer. Unfortunately, most of the antimicrobial surveillance systems only target specific strains or species and therefore are not well equipped for examining genes of drug resistance. To better understand the molecular carbapenem resistance mechanisms in CRKPN isolates in Chongqing, the carbapenemase genes in the 79 collected CRKPN isolates were detected. KPC-2 was found to be the most prevalent carbapenemase type in CRKPN (41/79,51.9\%), which was lower than that was reported by Qi Wang et al. (919/1201, 76.5\%) who enrolled CRKPN isolates from 25 provinces across China [11]. NDM, including NDM-1 (27/79, 34.2\%) and NDM-5 (4/79, 5.1\%), was the secondary most predominant carbapenemase type, the percentage of which were much higher than that was reported by Qi Wang et al. (NDM-1, 9.3\%; NDM-5, 1.7\%) [11]. It was especially noticeable that one CRKPN strain isolated from a patient with tumor co-expressed 3 types of carbapenemase genes $\left(b / a_{\mathrm{KPC}-2}, b / a_{\mathrm{NDM}-1}\right.$ and $\left.b / a_{\mathrm{IMP}-4}\right)$. To the best of our knowledge, there is only one study from Saudi Arabia that reported the first isolation of $K$. pneumoniae isolates co-harboring triple carbapenemase genes (including $b / a_{\mathrm{KPC}}, b / a_{\mathrm{NDM}-1}$ and $\left.b / a_{0 X A-48}\right)$. Among the 21 carbapenem-resistant $K$. pneumoniae isolates tested from Saudi Arabia, 17 strains carried triple resistant genes including $b / a_{\mathrm{KPC}}, b / a_{\mathrm{NDM}-1}$ and $b / a_{\mathrm{OXA}-48}$, while the other 4 carried double resistant genes $\left(b / a_{\mathrm{KPC}}\right.$ and $\left.b / a_{\mathrm{OXA}-48}\right)$ or $\left(b / a_{\mathrm{NDM}-1}\right.$ and $\left.b / a_{0 \times A-48}\right)[19]$.

As to the geographical distribution of the carbapenemase genes, bla $a_{\text {NDM }}$ was predominant in the middle of Chongqing. The differential compositions of carbapenemase gene types in different areas in Chongqing might due to nosocomial spread or unrational uses of anibiotics in different regions.

To our relief, the AMR of $P$. aeruginosa in Chongqing all showed decreasing tendencies, which was consistent with the results from CHINET [1]. Though significant decreases in resistance trends were also seen in $P$. aeruginosa to amikacin and gentamicin in a 10year study from 2005 to 2014 in the third largest tertiary healthcare university hospital in Serbia, significant increasing trends were witnessed in resistance rates to imipenem and meropenem [20]. In order to comprehensively understand the antibiotic resistance situation in $P$. aeruginosa, more detailed hierarchical researches of $P$. aeruginosa should be executed.

While important temporal decreases in susceptibility rates among $A$. baumannii complex isolates were observed for all the antimicrobial agents tested from medical centers enrolled in the SENTRY Program [21], our study demonstrated an approximate 10 percent increase of the annual resistance rates of $A$. baumannii complex to meropenem, which was similar to that of a multi-center study report by K. Dafopoulou, et al. in Greece, from 2010-2015 [3]. Nevertheless, nearly a ten percentage decrease in amikacin resistance rate was observed for $A$. baumannii in our study.

For $S$. aureus, MRSA isolation rates presented a slow decrease and sustained at lower levels below $30 \%$, which was different from the report of CHINET in China, which showed a significant decrease from $47.9 \%$ in 2012 to $35.3 \%$ in 2017 [1]. As for Enterococcus spp, the isolation rate of vancomycin resistant Enterococcus (VRE) maintained at a low level as compared with the result from CHINET [1].

Our study had several limitations. First, the AST results of tigecycline and polymyxin B were not included due to methodological defects employed. Second, the distribution map of the carbapenemase genes only cover fifteen districts out of the 38 districts in Chongqing due to economic burdens and strain preservation problems. Third, this was a retrospective observational study in Chongqing, and our results might not be applicable to other settings. In the future, more CRE isolates from other hospitals will be included so that we can understand the molecular mechanisms and their dynamic changes more comprehensively. 


\section{Conclusion}

In conclusion, our study provided a comprehensive provincial picture of the significant changes and trends in antimicrobial resistance of clinically important pathogens in Chongqing, which could be used to update the evidence-based guidelines on antimicrobial prescriptions in southwest, China. Although overall, many AMRs showed decreasing trends, further hierarchical researches should be executed according to different age groups, hospital wards, resistance degrees (MDR/XDR/PDR), and so on. To the best of our knowledge, this is the first report on a CRKPN isolate co-harboring triple carbapenemases including blaKPC-2, blaNDM-1 and blalMP-4 worldwide. At a time when novel and effective antibiotic compounds have not become available, containment of CPE is bound to rely on stricter infection control measures in hospitals.

\section{Abbreviations}

AMR: Antimicrobial resistance; BSIs: Bloodstream infections; CARSS: China Antimicrobial Resistance Surveillance System; CLSI: Clinical and Laboratory Standards Institute; CRABA: Carbapenem-resistant Acinetobacter baumannii; CRE: Carbapenem-resistant Enterobacteriaceae, CRECO: Carbapenem-resistant Escherichia coli; CRKPN: Carbapenemresistant Klebsiella pneumoniae; CRPAE: Carbapenem-resistant Pseudomonas aeruginosa; MIC: Minimum inhibitory concentration; MRSA: Methicillin-resistant Staphylococcus aureus, VREFM: Vancomycin-resistant Enterococcus faecium; WHO: World health organization

\section{Antimicrobials}

PEN: Penicillin G; AMP: Ampicillin; OXA: Oxacillin; PIP: Piperacillin; TZP: Piperacillin/Tazobactam; CZO: Cefazolin; CXM: Cefuroxime; CAZ: Ceftazidime; CRO: Ceftriaxone; FEP: Cefepime; ATM: Aztreonam; ETP: Ertapenem; IMP: Imipenem; MEM: Meropenem; CIP: Ciprofloxacin; LEV: Levofloxacin; MXF: Moxifloxacin; AMK: Amikacin; GEH: Gentamicin-High level; TOB: Tobramycin; ERY: Erythromycin; CLI: Clindamycin; CHL: Chloramphenicol; TCY: Tetracycline; LZD: Linezolid; VAN: Vancomycin

\section{Declarations}

\section{Acknowledgements}

We would like to thank all the enrolled laboratories for their participation in our study.

\section{Funding}

This study was supported in part by the National Natural Science Foundation of China (Grant No. 81772239 and 31500749), the Science and Technology Research Program of Chongqing Municipal Education Commission (Grant No. KJ1500235 and KJ1702022), and the Medical Research Program of Chongqing Health and Family Planning Commission (No.2017MSXM009, 2018MSXM009 and 2016MSXM001).

\section{Availability of data and materials}

All the data of this article is available from the corresponding author if reasonably requested.

\section{Authors' contributions}

All the authors contributed to data analysis, drafting and revising the article, gave final approval of the version to be published, and agree to be accountable for all aspects of the work. SS, SQN and SFH designed this study, participated in statistic calculations and interpretation of data, and drafted this manuscript. LY,CMZ and JDS collected the raw data of ARINCQ by Whonet 5.6.

\section{Ethics approval and consent to participate}

Not applicable.

\section{Consent for publication}

Not applicable. 


\section{Competing interests}

The authors declare that they have no competing interests.

\section{References}

1. Hu F, Zhu D, Wang F, Wang M. Current Status and Trends of Antibacterial Resistance in China. Clin Infect Dis. 2018; 67 (suppl_2): S128-S134.

2. Dickstein Y, Temkin E, Ish Shalom M, Schwartz D, Carmeli Y, Schwaber MJ. Trends in antimicrobial resistance in Israel, 20142017. Antimicrob Resist Infect Control. 2019; 8: 96.

3. Dafopoulou K, Tsakris A, Pournaras S. Changes in antimicrobial resistance of clinical isolates of Acinetobacter baumannii group isolated in Greece, 2010-2015. J Med Microbiol. 2018; 67(4): 496-8.

4. Goto M, McDanel JS, Jones MM, Livorsi DJ, OhI ME, Beck BF, et al. Antimicrobial Nonsusceptibility of Gram-Negative Bloodstream Isolates, Veterans Health Administration System, United States, 2003-20131. Emerg Infect Dis. 2017; 23(11):181525.

5. Hu FP, Guo Y, Zhu DM, Wang F, Jiang XF, Xu YC, et al. Resistance trends among clinical isolates in China reported from CHINET surveillance of bacterial resistance, 2005-2014. Clin Microbiol Infect. 2016; 22 Suppl 1: S9-14.

6. Musicha P, Cornick JE, Bar-Zeev N, French N, Masesa C, Denis B, et al. Trends in antimicrobial resistance in bloodstream infection isolates at a large urban hospital in Malawi (1998-2016): a surveillance study. Lancet Infect Dis. 2017; 17(10):104252.

7. Tian L, Zhang Z, Sun Z. Antimicrobial resistance trends in bloodstream infections at a large teaching hospital in China: a 20year surveillance study (1998-2017). Antimicrob Resist Infect Control. 2019; 8: 86.

8. Diekema DJ, Hsueh PR, Mendes RE, Pfaller MA, Rolston KV, Sader HS, Jones RN. The microbiology of bloodstream infection: 20-Year trends from the SENTRY Antimicrobial Surveillance Program. Antimicrob Agents Chemother. 2019; 63 (7). pii: e0035519.

9. Zhang J, Zhao C, Chen H, Li H, Wang Q, Wang Z, Zhang F, Wang H. A multicenter epidemiology study on the risk factors and clinical outcomes of nosocomial intra-abdominal infections in China: results from the Chinese antimicrobial resistance surveillance of nosocomial infections (CARES) 2007-2016. Infect Drug Resist. 2018, 11: 2311-9.

10. Hayajneh WA, Hajj A, Hulliel F, Sarkis DK, Irani-Hakimeh N, Kazan L, Badal RE. Susceptibility trends and molecular characterization of Gram-negative bacilli associated with urinary tract and intra-abdominal infections in Jordan and Lebanon: SMART 2011-2013. Int. J. Infect. Dis. 2015; 35: e56-e61

11. Wang Q, Wang X, Wang J, Ouyang P, Jin C, Wang R, et al. Phenotypic and Genotypic Characterization of Carbapenem-resistant Enterobacteriaceae: Data From a Longitudinal Large-scale CRE Study in China (2012-2016). Clin Infect Dis. 2018; 67 (suppl_2): S196-S205.

12. Raveh D, Rudensky B, Huerta M, Aviv Y, Yinnon AM. Use of time-trend analysis in the design of empirical antimicrobial treatment of urinary tract infection. Eur J Clin Microbiol Infect Dis 2003; 22:158-64.

13. Raveh D, Rudensky B, Schlesinger Y, Benenson S, Yinnon AM. Susceptibility trends in bacteraemias: analyses of 7544 patientunique bacteraemic episodes spanning 11 years (1990-2000). J Hosp Infect 2003; 55:196-203.

14. Kim B, Kim Y, Hwang H, Kim J, Kim SW, Bae IG, et al. Trends and correlation between antibiotic usage and resistance pattern among hospitalized patients at university hospitals in Korea, 2004 to 2012: A nationwide multicenter study. Medicine (Baltimore). 2018; 97(51): e13719.

15. Durante-Mangoni E, Andini R, Zampino R. Management of carbapenem resistant Enterobacteriaceae infections. Clin Microbiol Infect. 2019; 25(8):943-50.

16. Righi E, Peri AM, Harris PN, et al. Global prevalence of carbapenem resistance in neutropenic patients and association with mortality and carbapenem use: systematic review and meta-analysis. J Antimicrob Chemother. 2017; 72(3):668-77.

17. Amit S, Mishali H, Kotlovsky T, Schwaber MJ, Carmeli Y. Bloodstream infections among carriers of carbapenem-resistant Klebsiella pneumoniae: etiology, incidence and predictors. Clin Microbiol Infect. 2015; 21(1):30-4.

18. Márió Gajdács. The concept of an ideal antibiotic: implications for drug design. Molecules. 2019; 24(5): 892.

Page 14/17 
19. Khan MA, Mohamed AM, Faiz A, Ahmad J. Enterobacterial infection in Saudi Arabia: First record of Klebsiella pneumoniae with triple carbapenemase genes resistance. J Infect Dev Ctries 2019 30; 13(4):334-41.

20. Mladenovic-Antic S, Kocic B, Velickovic-Radovanovic R, Dinic M, Petrovic J, Randjelovic G, et al. Correlation between antimicrobial consumption and antimicrobial resistance of Pseudomonasaeruginosa in a hospital setting: a 10-year study. J Clin Pharm Ther. 2016; 41(5): 532-7.

21. Gales AC, Seifert H, Gur D, Castanheira M, Jones RN, Sader HS. Antimicrobial susceptibility of Acinetobacter calcoaceticusAcinetobacter baumannii complex and Stenotrophomonas maltophilia clinical isolates: results from the SENTRY Antimicrobial Surveillance Program (1997-2016). Open Forum Infect Dis. 2019; 6 (Suppl 1): S34-S46.

\section{Figures}

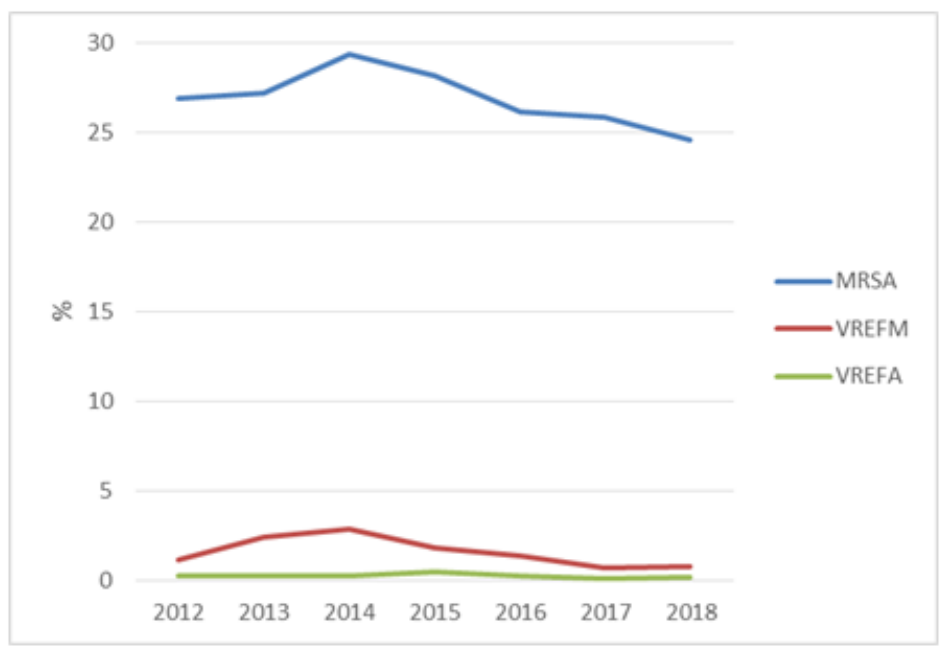

\section{Figure 1}

Isolation rates of MRSA, VREFM and VREFA from 2012-2018 MRSA, methicillin-resistant Staphylococcus aureus; VREFM, vancomycine-resistant Enterococcus faecium; VREFA, vancomycine-resistant Enterococcus faecalis

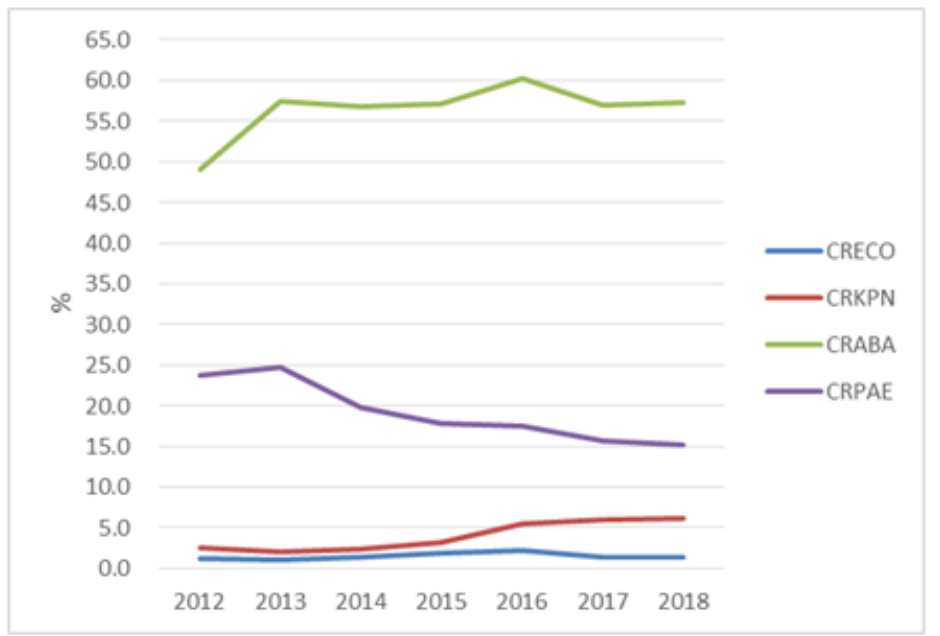

\section{Figure 2}

Isolation rates of CRECO, CRKPN, CRABA and CRPAE from 2012 to 2018 CRECO, carbapenem-resistant Escherichia coli; CRKPN, carbapenem-resistant Klebsiella pneumoniae; CRABA, carbapenem-resistant Acinetobacter baumannii; CRPAE, carbapenemresistant Pseudomonas aeruginosa 


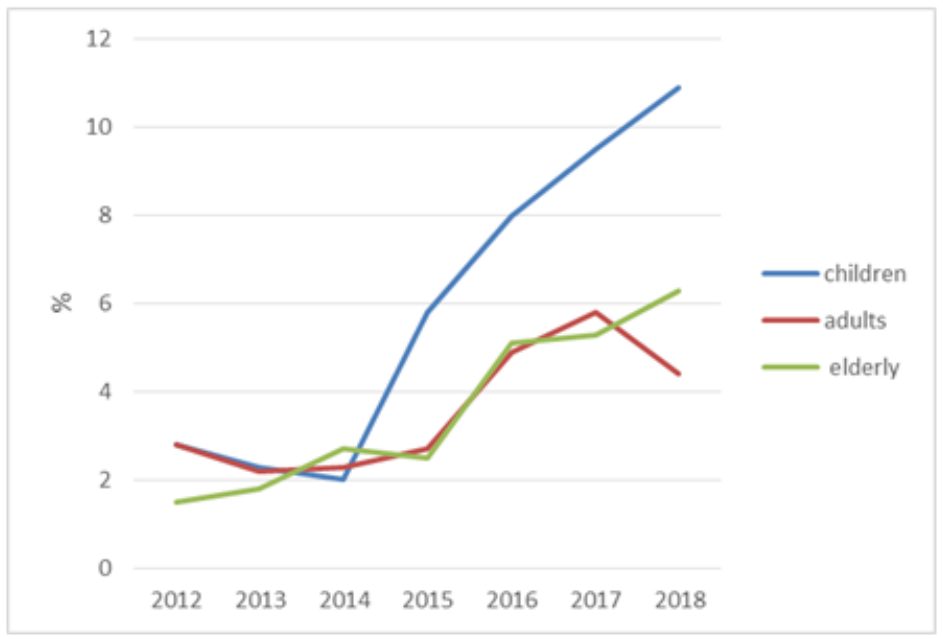

\section{Figure 3}

Isolation rates of CRKPN for different age groups from 2012 to 2018 CRKPN, carbapenem-resistant Klebsiella pneumoniae

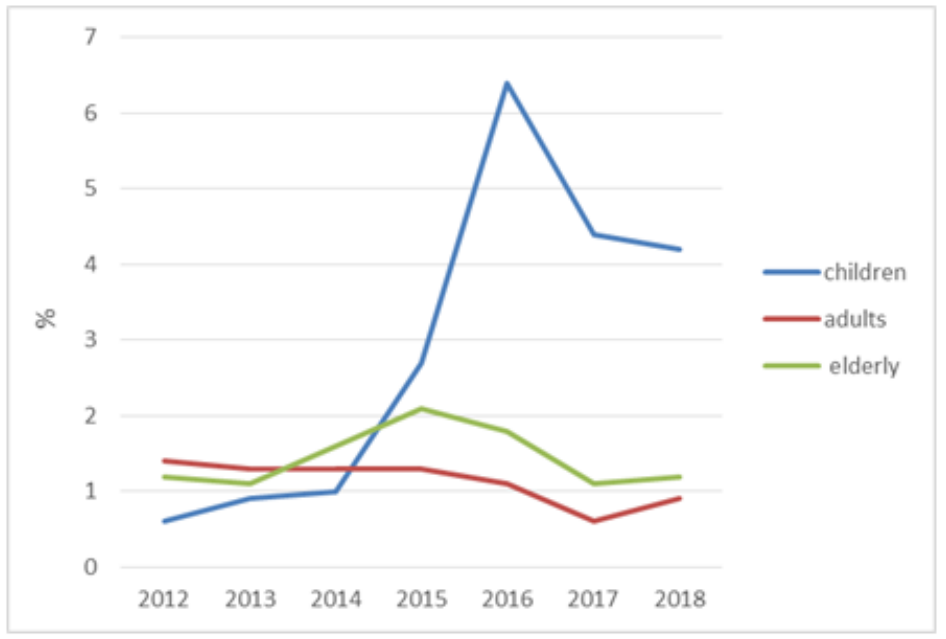

Figure 4

Isolation rates of CRECO for different age groups from 2012 to 2018 CRECO, carbapenem-resistant Escherichia coli 


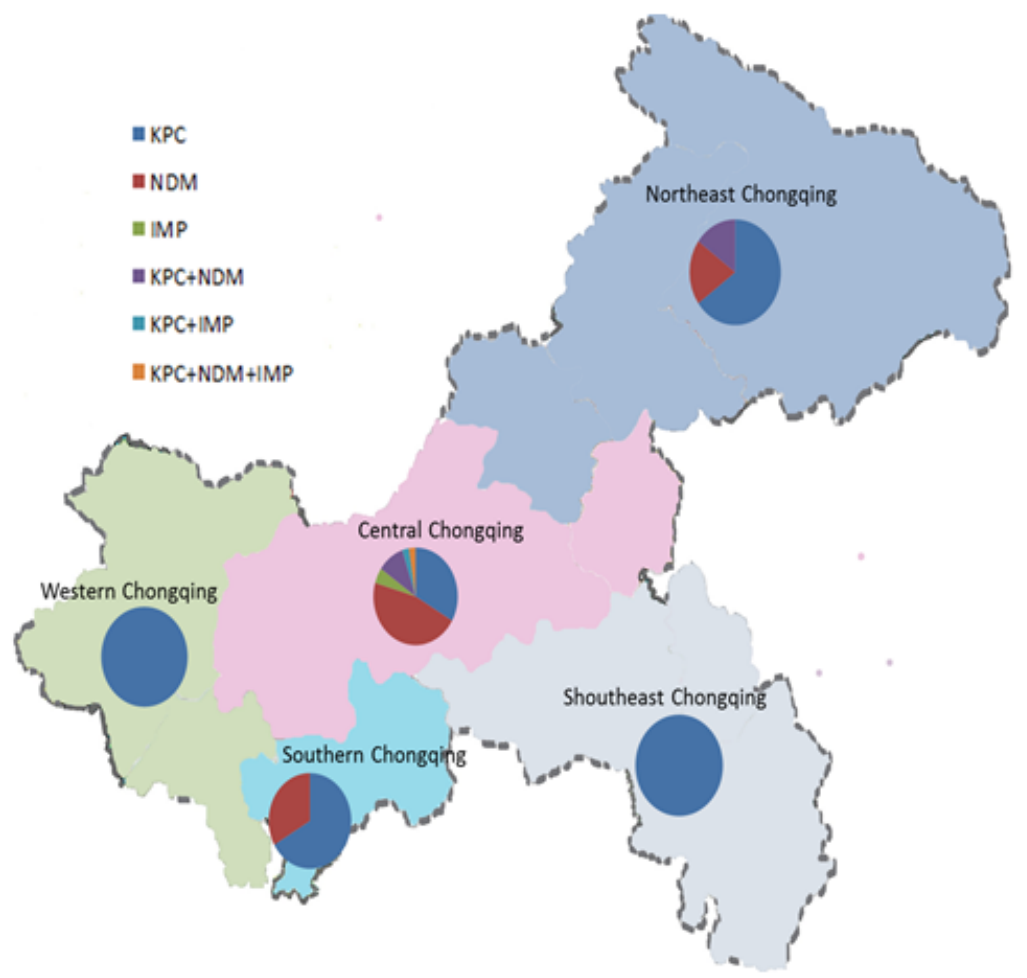

\section{Figure 5}

Distribution map of the carbapenemase genes from the CRKPN isolates in Chongqing CRKPN, carbapenem-resistant Klebsiella pneumon. Note: The designations employed and the presentation of the material on this map do not imply the expression of any opinion whatsoever on the part of Research Square concerning the legal status of any country, territory, city or area or of its authorities, or concerning the delimitation of its frontiers or boundaries. This map has been provided by the authors. 